\title{
Utilização do Internamento Hospitalar em Portugal Continental por Crianças com Doenças Crónicas Complexas (2011 - 2015)
}

\section{Hospital Inpatient Use in Mainland Portugal by Children with Complex Chronic Conditions (2011 - 2015)}

Resultados: Nos 419927 episódios, constavam códigos de doenças crónicas complexas em 64918 (15,5\%). Estes episódios com doenças crónicas complexas representaram $29,8 \%$ dos dias de internamento, $39,4 \%$ da despesa e $87,2 \%$ dos óbitos. Custaram o dobro dos episódios sem doenças crónicas complexas (€1467 vs €745) e tiveram uma mortalidade 40 vezes superior $(2,4 \%$ vs $0,06 \%)$. Do total, $46,0 \%$ foram programados (sem doenças crónicas complexas $23,2 \%$ ); $64,8 \%$ ocorreram em hospitais grupo III - IV (sem doenças crónicas complexas $27,1 \%$ ). Nos episódios com doenças crónicas complexas, a doença maligna foi a categoria mais frequente $(23,0 \%)$; a maior demora mediana (12 dias, 6 - 41), despesa mediana (€3568,929 - 24 602) e mortalidade $(13,4 \%)$ verificaram-se na categoria neonatais.

Discussão: Esta análise mostrou que, embora o número absoluto de internamentos de doentes pediátricos esteja a diminuir em Portugal Continental, à semelhança de outros países desenvolvidos, os internamentos com doenças crónicas complexas têm vindo a aumentar proporcionalmente, sendo mais prolongados, onerosos e com maior probabilidade de morte do que os episódios sem doenças crónicas complexas (tendências acentuadas quando constam duas ou mais doenças crónicas complexas).

Conclusão: As doenças crónicas complexas são relevantes na atividade e despesa do internamento hospitalar pediátrico em Portugal Continental. Este reconhecimento e a integração de cuidados paliativos pediátricos desde o diagnóstico são essenciais para adequar a utilização do hospital, desenvolvendo alternativas efetivas e sustentáveis que vão ao encontro das necessidades das crianças, famílias e profissionais.

Palavras-chave: Criança; Cuidados Paliativos; Determinação de Necessidades de Cuidados de Saúde; Hospitalização; Portugal

\section{ABSTRACT}

Introduction: Due to epidemiological change, interest in complex chronic conditions has been increasing within the pediatric health system. As such, we aim to evaluate hospital inpatient care in the National Health Service (mainland Portugal) by pediatric patients $(0-17$ years) with complex chronic conditions.

Material and Methods: Observational longitudinal retrospective epidemiological study using anonymized administrative data. We selected hospitalizations within the pediatric age limit, 2011 - 2015; healthy newborns and radiotherapy outpatients were excluded. A descriptive analysis of the admissions with complex chronic conditions was analysed by number of complex chronic conditions categories and by complex chronic conditions categories. Non-parametric tests were applied to length of stay, expense, and mortality. Results: Out of 419927 admissions, 64918 (15.5\%) contained at least one complex chronic conditions code. These admissions due to complex chronic conditions represented $29.8 \%$ of hospital days, $39.4 \%$ of expense and $87.2 \%$ of deaths. Compared to those without complex chronic conditions, expense was double (median €1467 vs €745) and mortality 40 times higher ( $2.4 \%$ vs $0.06 \%)$. Of these, $46 \%$ were planned (no complex chronic conditions $23.2 \%$ ); $64.8 \%$ occurred in group III - IV hospitals (no complex chronic conditions $27.1 \%)$. Malignant was the most frequent category (23.0\%); neonatal had the highest median length of stay (12 days, $6-41)$, median expense (€3568,929 - 24602$)$, and number of deaths (43.5\% of total).

Discussion: As in other developed countries where the number of pediatric admissions is decreasing, in mainland Portugal we found an increase in the proportion of complex chronic conditions admissions, which are longer, costlier and deadlier (trends intensified in the presence of two or more complex chronic conditions categories).

Conclusion: Complex chronic conditions are relevant in the activity and costs regarding pediatric hospitalizations in mainland Portugal. Recognizing this and integrating pediatric palliative care from the moment of diagnosis are essential to promote appropriate hospital

1. Observatório Português de Cuidados Paliativos. Instituto de Ciências da Saúde. Universidade Católica Portuguesa. Lisboa. Portugal.

2. Serviço de Pediatria. Instituto Português de Oncologia de Lisboa Francisco Gentil. Lisboa. Portugal.

3. Departamento de Pediatria. Hospital de Santa Maria. Centro Hospitalar Lisboa Norte. Lisboa. Portugal.

4. Serviço de Pediatria Médica. Departamento Pediátrico. Centro Hospitalar e Universitário de Coimbra. Coimbra. Portugal.

5. Departamento de Políticas e Gestão do Sistema de Saúde. Escola Nacional de Saúde Pública. Universidade NOVA de Lisboa. Lisboa. Portugal.

6. Centro de Investigação em Saúde Pública. Escola Nacional de Saúde Pública. Universidade NOVA de Lisboa. Lisboa. Portugal.

$\triangle$ Autor correspondente: Ana Forjaz Lacerda. alacerda@ipolisboa.min-saude.pt

Recebido: 20 de fevereiro de 2018 - Aceite: 15 de janeiro de 2019 | Copyright $\odot$ Ordem dos Médicos 2019 
use, through the development of effective and sustainable alternatives that meet the needs of children, families, and healthcare professionals.

Keywords: Child; Hospitalization; Needs Assessment; Palliative Care; Portugal

\section{INTRODUÇÃO}

Nos últimos anos temos assistido a um interesse crescente sobre a doença crónica nos sistemas de saúde, fruto da mudança epidemiológica iniciada nas últimas décadas. A Pediatria não é exceção, dado o aumento da sobrevivência de recém-nascidos e crianças com necessidades de saúde complexas e condições que anteriormente eram rapidamente fatais. ${ }^{1-4}$

Acresce que o atual cenário socioeconómico obriga a um repensar do sistema de saúde, que no século $X X I$ se quer racionalizado, proativo em vez de reativo, e centrado nas necessidades dos utentes e não na doença per se ou nas rotinas dos profissionais e instituições de saúde. ${ }^{5-7}$

Esta renovação é também indissociável da expansão da disciplina dos cuidados paliativos e do seu âmbito, agora na sua 'terceira vaga', não circunscritos apenas à fase terminal ou de fim de vida, ${ }^{8}$ que levou em 2014 à recomendação da Assembleia Geral das Nações Unidas para a integração plena destes cuidados nos sistemas de saúde dos seus Estados-Membros. ${ }^{9}$

Um dos primeiros requisitos para o desenvolvimento equitativo e sustentável de serviços é a avaliação das necessidades. ${ }^{10}$ Para analisar a prevalência de crianças medicamente complexas e com necessidade de cuidados paliativos pediátricos foi desenvolvida uma classificação — doenças crónicas complexas (DCC). ${ }^{11}$ Utilizada em estudos de mortalidade em vários países, ${ }^{3,12-14}$ permite também identificar as crianças com utilização intensiva dos serviços de saúde. ${ }^{15-18}$ Estas requerem um apoio coordenado de saúde e psicossocial desde o diagnóstico, dirigido igualmente à família e à comunidade,${ }^{6}$ um dos princípios básicos dos cuidados paliativos pediátricos. ${ }^{19} \dot{E}$ ainda relevante notar a dificuldade em traçar fronteiras entre crianças com doenças limitantes ou ameaçadoras da vida e crianças com incapacidades, ${ }^{20,21}$ e a consequente recomendação de organizar serviços equitativos e sustentáveis que cruzem essas fronteiras. ${ }^{4,20,21}$

Em Portugal, o alerta para esta modificação da realidade pediátrica é recente. Até 2013 não era oficialmente reconhecida atividade em cuidados paliativos pediátricos no país. ${ }^{22}$ Desde então, com a criação de dois grupos de trabalho, na Sociedade Portuguesa de Pediatria e na Associação Portuguesa de Cuidados Paliativos, o tema tem ganho visibilidade crescente. O relatório do grupo de trabaIho nomeado pelo Ministério da Saúde, publicado em 2015, recomenda a implementação estratégica de equipas e unidades de cuidados paliativos pediátricos. ${ }^{23}$ No entanto, até agora nenhum estudo foi feito em Portugal relativamente à frequência de crianças com necessidades paliativas, uma prioridade já reconhecida noutros países. ${ }^{20,24,25}$

É nesse sentido que surge este trabalho, que pretende avaliar a utilização do internamento hospitalar do Serviço Nacional de Saúde (SNS) em Portugal Continental pelas crianças com DCC, como ponto de partida para uma revisão do modelo de cuidados prestados.

\section{MATERIAL E MÉTODOS}

Este estudo epidemiológico, observacional longitudinal retrospetivo, analisa os episódios de internamento (doravante designados episódios) com DCC em instituições hospitalares do SNS em Portugal Continental, em idade pediátrica na admissão, ${ }^{26}$ durante cinco anos (1 janeiro 2011 - 31 dezembro 2015).

\section{Doenças crónicas complexas}

Dado o objetivo do trabalho, optámos por utilizar uma classificação desenvolvida em 2000 nos Estados Unidos da América (EUA) por Feudtner e colaboradores, que definiram DCC como "qualquer situação médica para que seja razoável esperar uma duração de pelo menos 12 meses (exceto em caso de morte) e que atinja vários diferentes sistemas ou um órgão de forma suficientemente grave, requerendo cuidados pediátricos especializados e provavelmente algum período de internamento num centro médico terciário". ${ }^{11}$

Esta classificação não só é facilmente operacionalizável e baseada em códigos da International Classification of Diseases (ICD), como é ampla e flexível, colocando o foco não apenas na duração ou gravidade da doença, mas também na utilização dos cuidados de saúde. A lista foi recentemente atualizada e expandida, incorporando agora códigos neonatais e outros indicativos de dependência de tecnologia ou transplantação de órgão. ${ }^{16}$

Nesta última versão, as DCC dividem-se em dez categorias: neurológicas / neuromusculares, cardiovasculares, respiratórias, renais / urológicas, gastrointestinais, hematológicas / imunológicas, metabólicas, outras congénitas / genéticas, malignas e neonatais. Inclui ainda dois domínios - um para dependência de tecnologia e outro para transplantação (que aparecem separados das categorias quando não classificados em uma delas - crianças com 0 categorias).

\section{Fontes de dados}

Em Portugal Continental são colhidos por rotina os dados dos internamentos hospitalares, alimentando a base de dados de morbilidade hospitalar da Administração Central do Sistema de Saúde (ACSS). Esta cede os dados anonimizados à Escola Nacional de Saúde Pública (ENSP-NOVA).

Da base de dados retirou-se informação sobre características demográficas do utente (idade, freguesia de residência), datas de admissão e alta, tipo de admissão (urgente ou programada), hospital, diagnósticos e procedimentos [codificados pela Classificação Internacional de Doenças - nona versão - Modificações Clínicas (CID-9-MC); 
número máximo de 59 e 51 por episódio, respetivamente], GDH (grupos de diagnósticos homogéneos), duração do internamento e destino após a alta.

\section{Definição da população em estudo}

Para este estudo selecionámos os episódios em que o utente tinha idade entre 0 e 17 anos e 364 dias, considerando para o efeito o menor valor no ano do episódio. Ao longo do texto, para simplificar a leitura, utilizámos a designação 'criança' para nos referirmos a estes utentes em idade pediátrica.

Excluímos os episódios de recém-nascidos vivos (diagnóstico principal CID-9-MC V30-V39), com alta vivo e duração de internamento igual ou inferior a três dias ( $n=270$ 572), assim como os episódios de administração ambulatória de radioterapia (diagnóstico principal CID-9-MC V580, 0 dias de internamento e sem códigos CID-9-CM que a portaria dos $\mathrm{GDH}$ define como necessários para serem considerados ambulatório, $\mathrm{n}=8061$ ).

\section{Definição de outras variáveis}

Quando a variável freguesia de residência tomava valores diferentes ao longo do ano, atribuímos o valor registado no primeiro episódio do ano. O concelho de residência foi identificado com base na freguesia e agregado em 'região de residência' segundo a NUTS II (Norte, Centro, Área Metropolitana de Lisboa, Alentejo, Algarve e Regiões Autónomas). ${ }^{27}$ Nas análises relativas a local de residência excluímos os episódios em que não foi possível atribuir região (no total: 1897, 0,5\%; com DCC: $645,1,0 \%$ ).

Baseámos a categorização dos 49 hospitais do SNS na Portaria $n^{\circ} 82 / 2014$, que classifica as instituições em quatro grupos, de acordo com a natureza das suas responsabilidades e o quadro das valências exercidas: grupo I (menor complexidade, área de influência direta), grupo II (complexidade intermédia, área de influência direta e indireta), grupo III (maior complexidade, área de influência direta e indireta) e grupo IV (especializados — Oncologia, Reabilitação e Saúde Mental). Alguns hospitais foram excluídos por não constarem desta Portaria e representarem um número residual de episódios (no total: quatro hospitais, 0,03\% dos episódios; com DCC: dois hospitais, 0,01\% dos episódios).

Para estimativa da despesa associada aos episódios, com base no preço do GDH atribuído, utilizámos a Portaria aplicável ao último ano em estudo (Portaria $n^{\circ}$ 234/2015).

A fim de enriquecer a análise, e porque outros autores descreveram já que a combinação de DCC representa uma maior complexidade de cuidados, ${ }^{15,17,18}$ optámos por distinguir dois grupos de crianças com DCC: com uma DCC ou com duas ou mais DCC.

\section{Autorizações}

Dado o desenho do estudo e a cedência dos dados anonimizados à ENSP-NOVA para investigação, não foi necessário solicitar autorização à Comissão de Ética e/ou à Comissão Nacional de Proteção de Dados.

\section{Análise}

Na primeira parte do estudo considerámos o universo do internamento hospitalar em Pediatria. Os episódios foram caracterizados por região de residência, tipo de admissão e grupo hospitalar. Para aferir se existiam diferenças significativas entre os episódios com e sem DCC, utilizámos o teste da mediana (duração de internamento e despesa, distribuições não normais) e o teste do qui-quadrado (mortalidade). Analisámos de forma descritiva a evolução anual da frequência, dias de internamento, despesa e óbitos dos episódios com DCC (em valor absoluto e proporcional do total dos episódios de internamento hospitalar em Pediatria).

$\mathrm{Na}$ segunda parte do estudo aprofundámos a caracterização dos episódios com DCC. Apurámos a sua distribuição por número de DCC e por categoria de DCC, analisando depois, para cada um destes grupos, as frequências absolutas e relativas por ano, região de residência, tipo de admissão e grupo hospitalar. De forma a perceber as diferenças associadas à presença de duas ou mais DCC, comparámos este grupo de doentes pediátricos com os restantes, em relação aos dias de internamento e despesa (com teste da mediana) e mortalidade (com teste do qui-quadrado).

Os dados foram analisados com os programas SPSS v24 ${ }^{\circledR}$ e Excel $2011^{\circledR}$.

\section{RESULTADOS}

O número total de internamentos hospitalares em idade pediátrica nos cinco anos estudados foi de 419927 (Tabela 1). Em 64918 episódios (15,5\%) constava pelo menos um código de DCC. Em Portugal Continental, o maior número de episódios com DCC verificou-se em crianças residentes na região Norte (23 194) e o menor no Algarve (2839); no entanto, a maior percentagem de episódios com DCC ocorreu na Área Metropolitana de Lisboa $(18,7 \%)$ e a menor na região Centro $(12,8 \%)$. Os episódios de crianças residentes nas Regiões Autónomas são apenas aqueles em que houve necessidade de transferência para instituições hospitalares mais diferenciadas no território Continental, não sendo os números representativos de todos os seus internamentos. Quase metade $(46,0 \%)$ dos episódios com DCC foram admissões programadas (o dobro dos episódios sem DCC, 23,2\%), ocorrendo a maioria $(64,8 \%)$ nos hospitais mais diferenciados dos grupos III e IV (vs $27,1 \%$ dos episódios sem DCC). Comparativamente com os episódios sem DCC, os episódios com DCC foram significativamente $(p<0,01)$ mais prolongados (mediana 5 vs 3 dias) e mais onerosos (mediana $€ 1467$ vs $€ 745$ ). Apresentaram igualmente uma maior percentagem de óbitos $(2,4 \%$ vs $0,06 \% ; p<0,01)$.

Nos episódios com DCC, observou-se ainda uma maior frequência de episódios de transferências para outras instituições hospitalares $(6,1 \%$ vs $2,1 \%)$.

A percentagem de episódios com DCC nos internamentos pediátricos aumentou entre 2011 e 2015 de $14,9 \%$ para $16,1 \%$ do total (Fig. 1). Neste período, os episódios 
Tabela 1 - Episódios dos doentes pediátricos, com e sem doenças crónicas complexas, no internamento hospitalar, Portugal Continental, $2011-2015$

\begin{tabular}{|c|c|c|c|c|c|}
\hline & Total & Sem DCC & & Com DCC & \\
\hline & $\mathbf{N}$ & $\mathbf{N}$ & $\mathbf{N}$ & Em \% do total & $\begin{array}{c}\text { Em \% dos episódios } \\
\text { com DCC }\end{array}$ \\
\hline Total & 419927 & 355009 & 64918 & $15,5 \%$ & $100,0 \%$ \\
\hline $\begin{array}{l}\text { Região de residência } \\
\text { Alentejo } \\
\text { Algarve } \\
\text { Área Metropolitana de Lisboa } \\
\text { Centro } \\
\text { Norte } \\
\text { Regiões Autónomas }\end{array}$ & $\begin{array}{r}27792 \\
20899 \\
110933 \\
99124 \\
157662 \\
1620\end{array}$ & $\begin{array}{r}24014 \\
18060 \\
90215 \\
86449 \\
134468 \\
551\end{array}$ & $\begin{array}{r}3778 \\
2839 \\
20718 \\
12675 \\
23194 \\
1069\end{array}$ & $\begin{array}{l}13,6 \% \\
13,6 \% \\
18,7 \% \\
12,8 \% \\
14,7 \% \\
66,0 \%\end{array}$ & $\begin{array}{r}5,8 \% \\
4,4 \% \\
31,9 \% \\
19,5 \% \\
35,7 \% \\
1,6 \%\end{array}$ \\
\hline $\begin{array}{l}\text { Tipo de admissão } \\
\text { Urgente } \\
\text { Programada }\end{array}$ & $\begin{array}{l}307828 \\
112099\end{array}$ & $\begin{array}{r}272742 \\
82267\end{array}$ & $\begin{array}{l}35086 \\
29832\end{array}$ & $\begin{array}{l}11,4 \% \\
26,6 \%\end{array}$ & $\begin{array}{l}54,0 \% \\
46,0 \%\end{array}$ \\
\hline $\begin{array}{l}\text { Grupo hospitalar } \\
\text { Grupo I } \\
\text { Grupo II } \\
\text { Grupo III } \\
\text { Grupo IV }\end{array}$ & $\begin{array}{r}184868 \\
96805 \\
129191 \\
8951\end{array}$ & $\begin{array}{r}172239 \\
86612 \\
95583 \\
468\end{array}$ & $\begin{array}{r}12629 \\
10193 \\
33608 \\
8483\end{array}$ & $\begin{array}{r}6,8 \% \\
10,5 \% \\
26,0 \% \\
94,8 \%\end{array}$ & $\begin{array}{l}19,5 \% \\
15,7 \% \\
51,8 \% \\
13,1 \%\end{array}$ \\
\hline $\begin{array}{l}\text { Dias de internamento } \\
\text { Mediana }(A I Q)\end{array}$ & $4(2-6)$ & $3(2-5)$ & $5(2-10)$ & - & - \\
\hline $\begin{array}{l}\text { Despesa }(€) \\
\text { Mediana (AIQ) }\end{array}$ & $811(579-1297)$ & $745(575-1112)$ & $1467(880-2666)$ & - & - \\
\hline $\begin{array}{l}\text { Mortalidade } \\
\text { Óbitos }\end{array}$ & 1765 & 226 & 1539 & $87,2 \%$ & $2,4 \%$ \\
\hline
\end{tabular}

AIQ: amplitude interquartis; DCC: doenças crónicas complexas. Na amplitude interquartis apresenta-se entre parênteses o valor do primeiro e terceiro quartis. Na análise por região de residência não são apresentados os valores desagregados dos episódios sem residência válida $\left(0,5 \%\right.$ do total; $1,0 \%$ dos episódios com DCC). Grupos hospitalares definidos segundo a Portaria $n^{\circ} 84 / 2014$. Na análise por grupo hospitalar não são apresentados os valores desagregados dos episódios em hospitais sem classificação quanto ao tipo $(0,03 \%$ do total; 0,01\% dos episódios com DCC). Despesa estimada segundo a Portaria $n^{\circ}$ 234/2015.

com DCC corresponderam em média a $29,8 \%$ dos dias de internamento, a $39,4 \%$ da despesa e a $87,2 \%$ dos óbitos. A representatividade dos episódios com DCC aumentou ao longo do tempo para os dias de internamento (de 28,0\% para $30,4 \%$ ) e para a despesa (de $38,0 \%$ para $40,8 \%$ ). Quanto à mortalidade, os valores foram de $86,5 \%$ no início do período (2011) e 86,0\% no fim (2015).

Esta tendência para a valorização proporcional da DCC verificou-se apesar de, no período em estudo, se ter observado nos episódios com DCC uma redução absoluta da frequência (13 325 para 12 750), dias de internamento (de aproximadamente $141 \mathrm{mil}$ para $131 \mathrm{mil}$ ) e óbitos (de 360 para 265) (Fig. 2). O valor da despesa manteve-se entre 47,9 e 49,5 milhões de euros.

Analisando apenas os episódios com DCC, a maioria (51 301; 79,0\%) apresentava apenas uma categoria (Fig. 3, Tabela 2). Em 1,1\% (715) não constavam códigos de categorias de DCC, mas apenas dos domínios dependência de tecnologia ou transplantação (0 categorias). A maioria destes episódios com 0 categorias de DCC ocorreram em crianças residentes na região Norte $(55,1 \%)$ e foram programados $(61,3 \%)$.

A percentagem de episódios em hospitais do Continente de crianças residentes nas Regiões Autónomas foi maior quando estavam presentes pelo menos duas categorias de DCC comparativamente à presença de 1 categoria e 0 categorias $(2,3 \%$ vs $1,5 \%$ e $0,8 \%$ ) (Tabela 2$)$.
Quando existiam duas ou mais categorias de DCC, a demora mediana foi significativamente maior ( 7 dias vs 4 dias e 4 dias, $p<0,01$ ), tal como a despesa mediana (€2058 vs €1344 e €1573, $p<0,01$ ) e a percentagem de episódios em que se verificou o óbito $(4,7 \%$ vs $1,8 \%$ e $0,0 \%, p<0,01$ ) (Tabela 2).

De entre as 10 categorias de DCC predominou a doença maligna (Tabela 2), correspondendo a $23,0 \%$ dos episódios. Seguiram-se as neurológicas / neuromusculares (17,6\%), cardiovasculares $(15,4 \%)$, renais / urológicas $(13,1 \%)$, hematológicas / imunológicas $(12,4 \%)$, outras anomalias congénitas / genéticas $(11,6 \%)$, gastrointestinais $(9,9 \%)$, neonatais $(7,7 \%)$, metabólicas $(7,3 \%)$ e respiratórias $(5,6 \%)$. A soma destas percentagens é superior a $100 \%$, já que ao mesmo episódio pode estar atribuída mais do que uma categoria.

A distribuição regional das 10 categorias de DCC seguiu o padrão geral (com pouco mais de um terço dos episódios na zona Norte), à exceção das categorias outras anomalias congénitas / genéticas (42,6\% na região Norte), hematológicas / imunológicas (47,7\% na Área Metropolitana de Lisboa) e metabólicas (49,0\% na região Norte).

Os episódios da categoria maligna foram os mais frequentemente programados $(69,1 \%)$. A maior percentagem de admissões urgentes $(76,7 \%)$ verificou-se nos episódios associados à categoria neonatais.

Mais de metade $(55,8 \%)$ dos episódios com DCC categoria doença maligna verificaram-se em hospitais do grupo IV. 


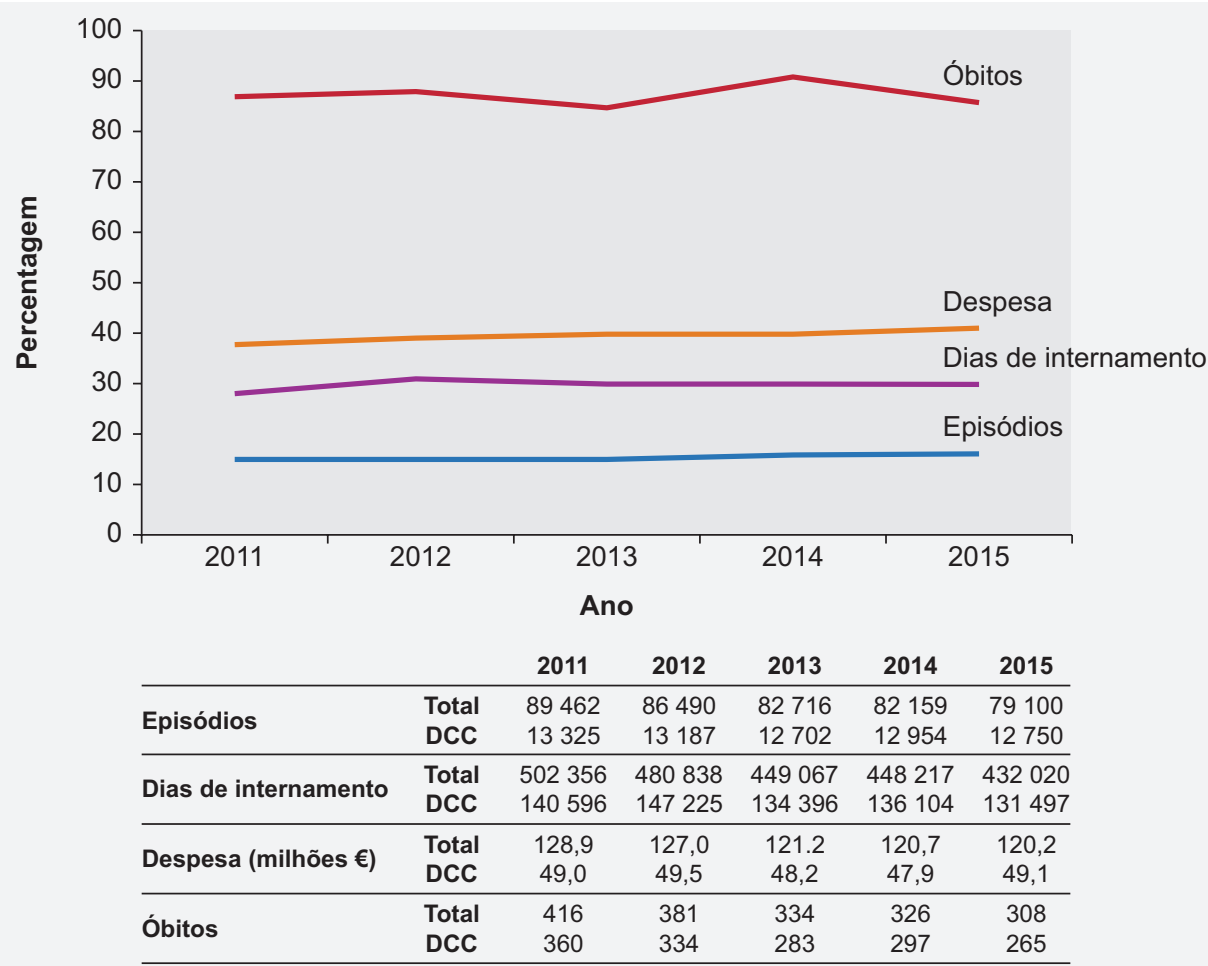

Figura 1 - Evolução anual das percentagens de episódios, dias de internamento, despesa e óbitos dos doentes pediátricos com doenças crónicas complexas no internamento hospitalar, Portugal Continental, 2011 - 2015

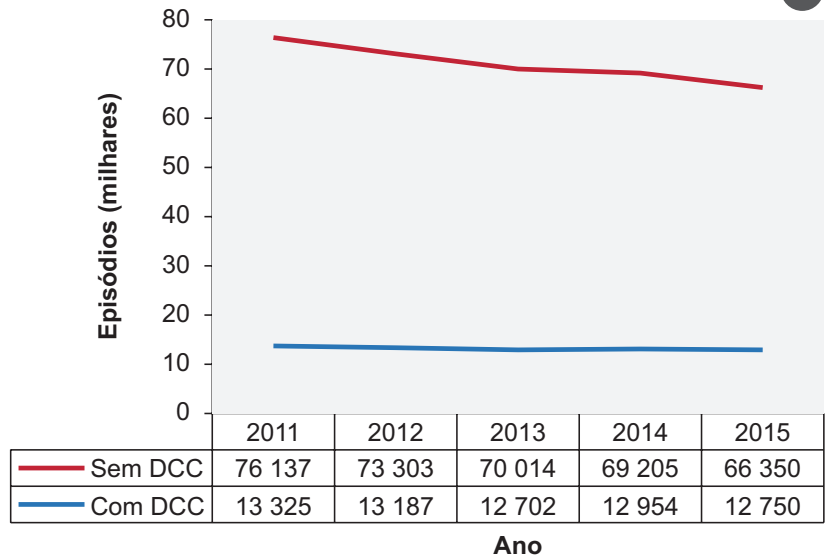

C

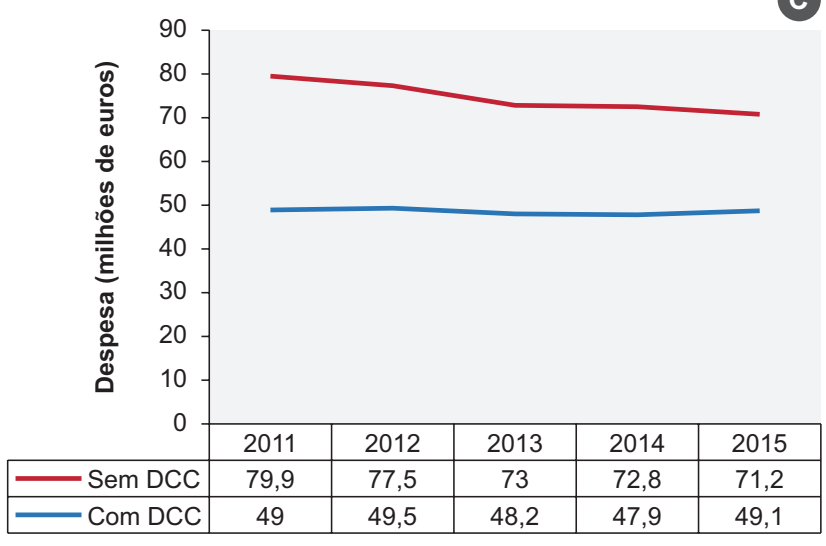

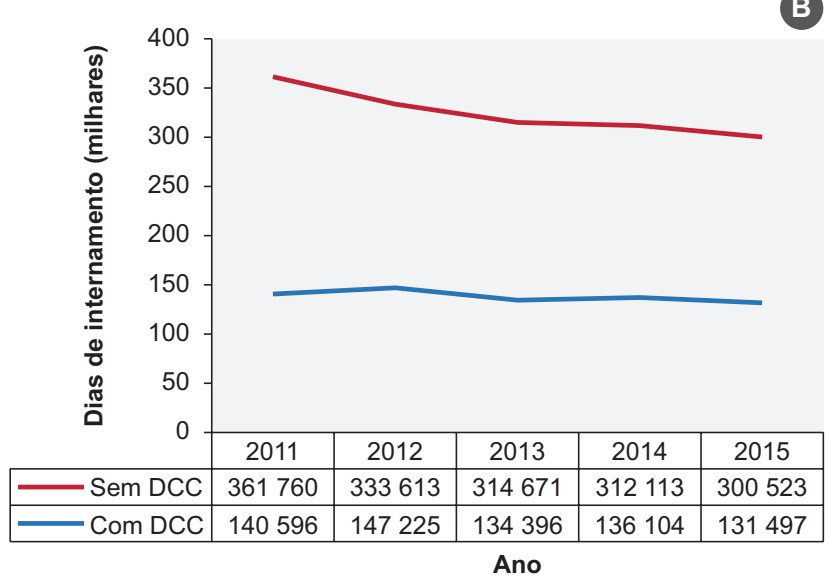

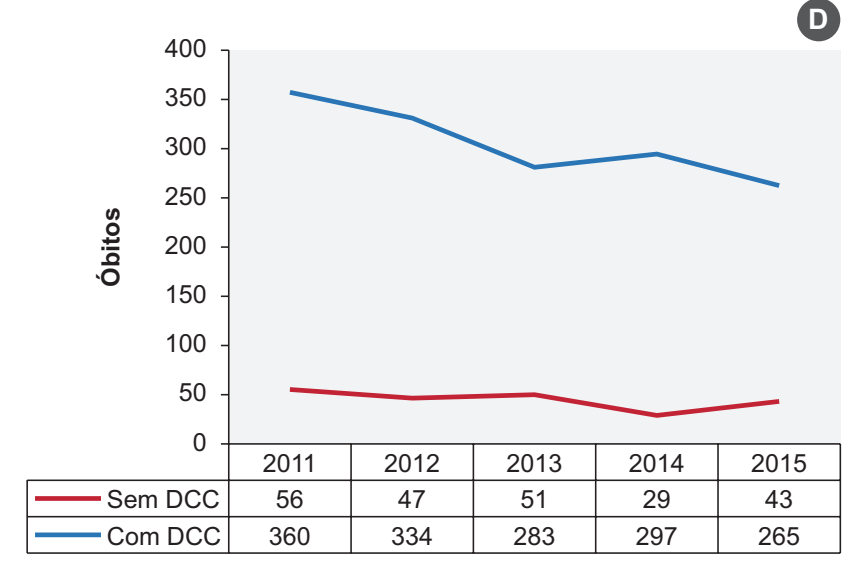

Figura 2 - Evolução anual dos episódios (A), dias de internamento (B), despesa (C) e óbitos (D), dos doentes pediátricos, com e sem doenças crónicas complexas, no internamento hospitalar, Portugal Continental, $2011-2015$ 


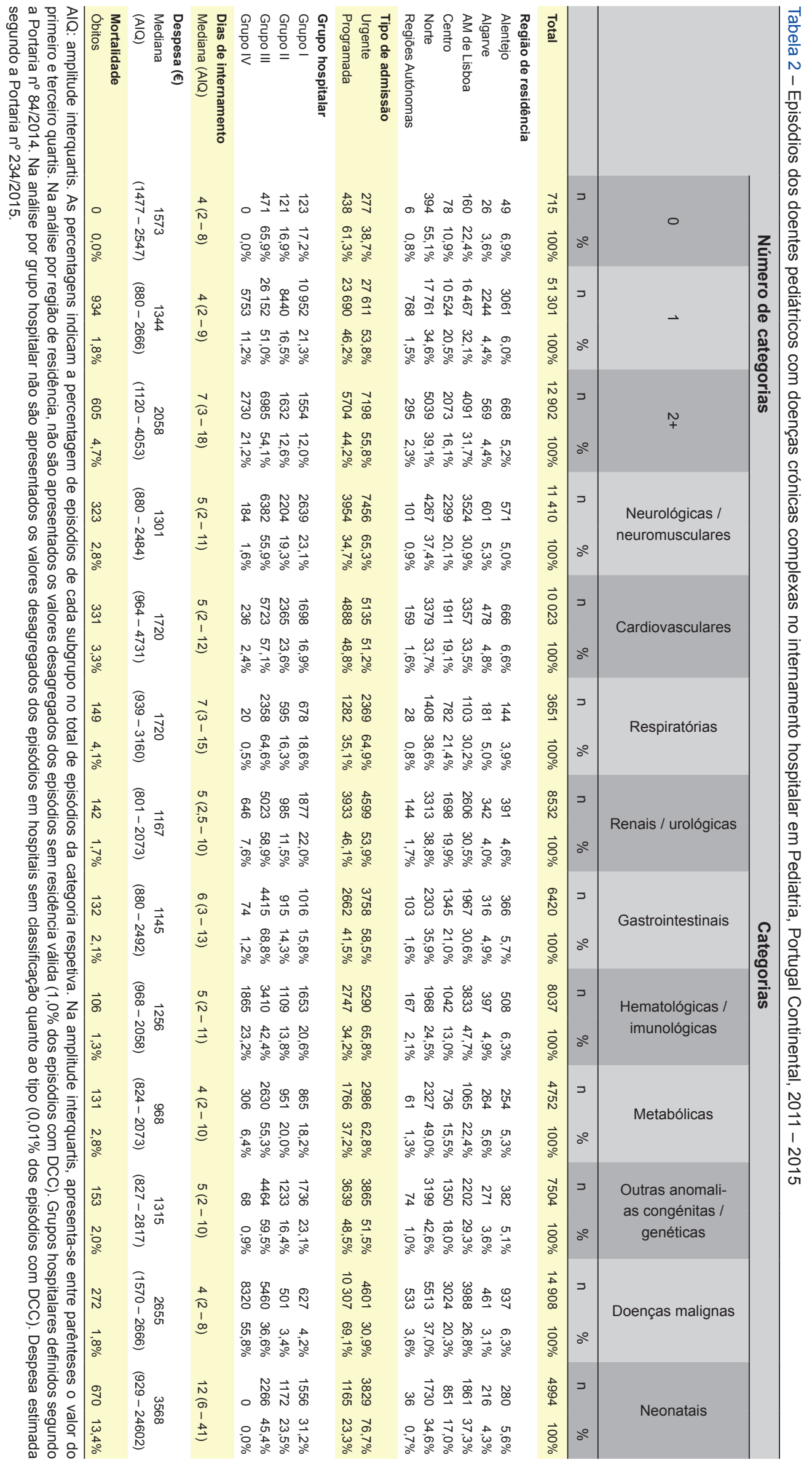


A maioria dos episódios das outras categorias de DCC ocorreram em hospitais do grupo III, variando entre $42,4 \%$ (hematológicas / imunológicas) e 68,8\% (gastrointestinais).

A duração dos episódios foi mais prolongada na categoria neonatais (mediana: 12 dias), tendo-se registado os episódios mais curtos nas categorias metabólicas e doenças malignas (mediana: 4 dias).

A despesa mediana variou entre $€ 968$ (metabólicas) e $€ 3568$ (neonatais). As outras categorias com despesa superior à mediana dos episódios com DCC (€1467) foram as de doença maligna (€2655), cardiovasculares e respiratórias (€1720).

A maior mortalidade registou-se nas categorias neonatais $(13,4 \%)$, respiratórias $(4,1 \%)$ e cardiovasculares $(3,3 \%)$.

Entre 2011 e 2015, verificou-se uma tendência crescente da proporção relativa das categorias respiratórias, outras anomalias congénitas / genéticas, neurológicas / neuromusculares, hematológicas / imunológicas, metabólicas e gastrointestinais. Em sentido contrário encontram-se as categorias doenças malignas, cardiovasculares, renais / urológicas e neonatais (Fig. 3).

\section{DISCUSSÃO}

O presente trabalho é o primeiro estudo relativo à utilização do internamento hospitalar por crianças com DCC em Portugal Continental e um dos primeiros estudos internacionais a utilizar para este fim a nova versão expandida dos códigos de DCC. ${ }^{16}$

Esta análise da base nacional de dados hospitalares entre 2011 - 2015 mostrou que, embora o número absoluto de internamentos de doentes pediátricos esteja a dimi- nuir, os internamentos de crianças com DCC têm vindo a aumentar proporcionalmente, sendo mais prolongados, onerosos e com maior probabilidade de ocorrência de morte do que os restantes. Estas diferenças acentuam-se quando se regista mais do que um código de DCC. Um estudo recente sobre o internamento pediátrico de curta duração (serviço de urgência) numa unidade hospitalar terciária nacional mostrara já que as crianças com doença crónica, representando 19,9\% dos episódios, permanecem internadas mais tempo e é mais provável serem transferidas para a enfermaria. ${ }^{28}$

Neste estudo, os 15,5\% de episódios com códigos de DCC corresponderam a $29,8 \%$ dos dias de internamento, a $39,4 \%$ da despesa e a $87,2 \%$ dos óbitos. Numa amostra de hospitais americanos em 2006 as DCC constituíram 10\% das admissões e representaram $25 \%$ dos dias de internamento, $40 \%$ dos gastos e $43 \%$ das mortes. ${ }^{15}$ No estudo que serviu de base à revisão da classificação de DCC (EUA, 2009 - 2010) a percentagem de episódios com DCC foi de $11,6 \%{ }^{16} \mathrm{~A}$ maior percentagem de episódios e de óbitos hospitalares em Portugal Continental poderá dever-se à falta de alternativas a estes cuidados, nomeadamente no domicílio e na comunidade.

Outros autores têm também reportado um peso crescente das DCC na utilização dos recursos hospitalares, ${ }^{15,17,18}$ o que se pode dever a múltiplos fatores. Em primeiro lugar, ao aumento da sobrevivência das crianças com $\mathrm{DCC}^{3}$ e ao aumento da complexidade ao longo do seu crescimento. ${ }^{15,29}$ Segundo, o número de episódios com DCC pode aumentar pela diminuição da duração dos internamentos, com períodos intercalados de cuidados domiciliários ${ }^{17}$ mas com readmissões frequentes dependentes das necessidades
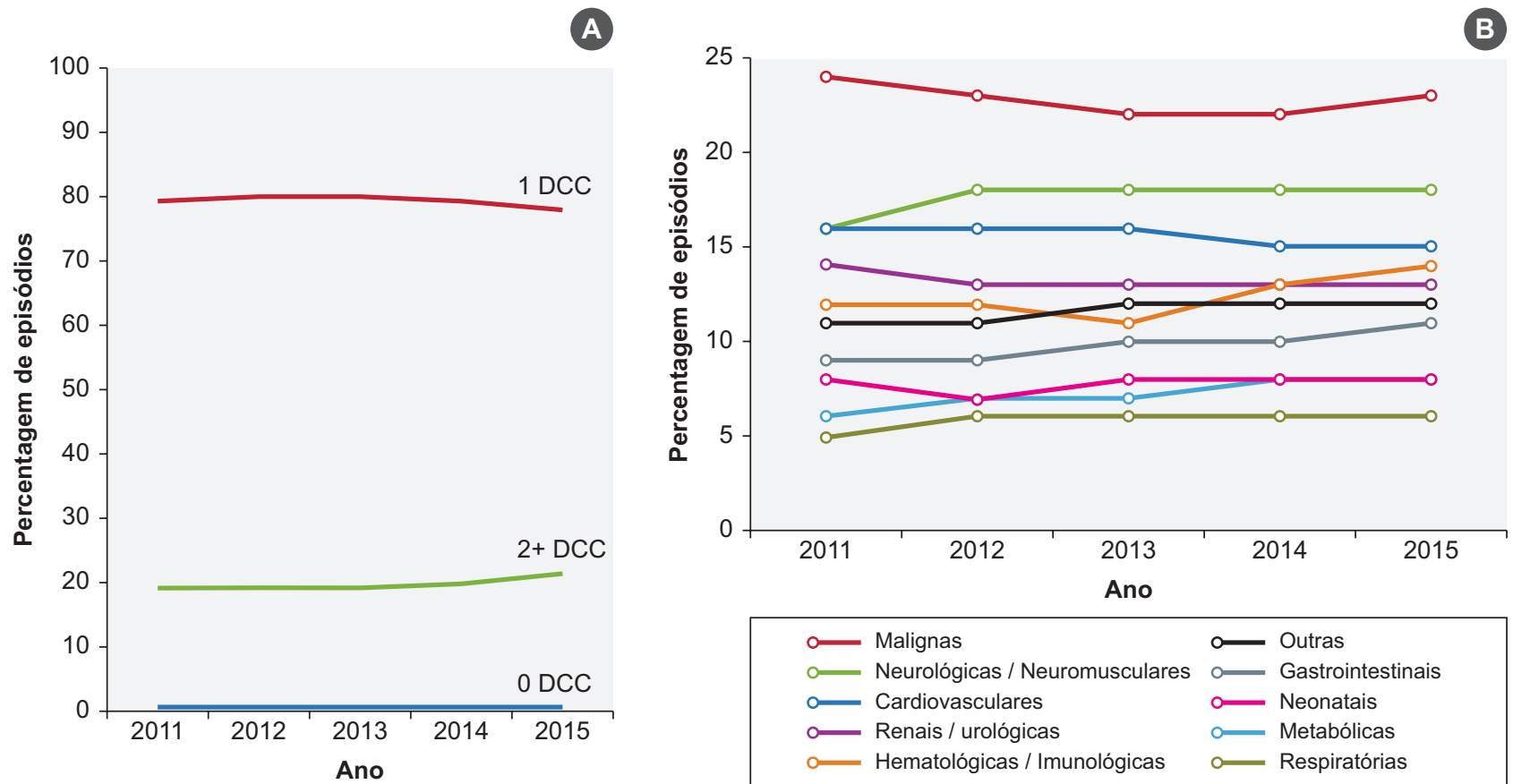

Figura 3 - Evolução anual da percentagem de episódios dos doentes pediátricos com doenças crónicas complexas no internamento hospitalar, Portugal Continental, 2011 - 2015. (A) Por número de categorias de doenças crónicas complexas; (B) por categorias de doenças crónicas complexas. 
assistenciais ${ }^{30}$ e provavelmente da ausência de resposta fora do hospital. ${ }^{18,31}$ Adicionalmente, a fragmentação e falta de coordenação entre prestadores no sistema de saúde, agravadas quando existe dependência de tecnologia(s), constituem fatores desestabilizadores para as crianças e famílias, ${ }^{32}$ resultando em readmissões mais frequentes e estadias mais longas do que o desejável. ${ }^{6,33}$

Terceiro, os cuidados de patologias mais frequentes e menos graves e complexas podem estar a ser deslocados para o ambulatório. ${ }^{15,29}$ De facto, em Portugal Continental ao longo dos anos estudados diminuíram o número e a duração de internamento de episódios sem DCC.

Apesar do maior número absoluto de episódios com DCC se ter registado na região Norte, a maior proporção de episódios com DCC verificou-se na Área Metropolitana de Lisboa. Verificámos também que a maioria dos episódios devidos a outras anomalias congénitas / genéticas e a doenças metabólicas, assim como aos domínios dependência de tecnologia ou transplantação ocorreu em crianças residentes na região Norte, enquanto quase metade dos episódios de doenças hematológicas / imunológicas se registaram em crianças residentes na Área Metropolitana de Lisboa. Estes resultados podem dever-se a diferenças na prevalência regional de DCC ou a uma diferente disponibilidade de cuidados, nomeadamente para o diagnóstico ou o acompanhamento ambulatório.

O facto de dois terços dos episódios relativos a crianças residentes nas Regiões Autónomas incluírem códigos DCC (e a maior percentagem de duas ou mais categorias, $27,6 \%$ ) reflete a necessidade da sua transferência para centros especializados ou com maior diferenciação (como é o caso das doenças malignas e hematológicas / imunológicas).

Enquanto a maioria dos episódios com DCC ocorreu em hospitais dos grupos III e IV (64,8\%), quase metade dos episódios sem DCC (48,5\%) verificou-se em hospitais do grupo I. Esta disparidade provavelmente reflete a diferente complexidade das situações clínicas com e sem DCC.

Em Portugal Continental, no internamento hospitalar pediátrico as DCC que predominam são as doenças malignas, ao contrário dos EUA, onde prevalecem as cardiovasculares e neurológicas / neuromusculares. ${ }^{16}$ Tal pode dever-se ao facto de neste país se promoverem os tratamentos e cuidados de suporte a crianças com cancro em ambulatório e no domicílio (EUA, 2003). ${ }^{34}$

Ainda nos EUA, a percentagem de internamentos, a duração e a despesa têm vindo a aumentar para todas as categorias de DCC exceto hematológicas / imunológicas. ${ }^{15}$ No nosso estudo não avaliámos esta evolução, dado o curto horizonte temporal. Será interessante fazê-lo futuramente, até porque a evidência é contraditória, já que outro estudo defende que as crianças com doenças hematológicas / imunológicas provavelmente têm menos alternativas de cuidados fora do hospital, sobretudo no último ano de vida. ${ }^{29}$

Como esperado, a maioria dos episódios relativos a crianças com doença maligna ou com dependência de tecnologia ou transplantação foram programados, para tratamentos ou intervenções previstas (provavelmente daí também estas crianças terem os internamentos mais curtos).

Foi ainda na doença maligna e nas hematológicas / imunológicas que se verificou a maior percentagem de internamentos em hospitais do grupo IV, o que se justifica pelo facto de aqui estarem incluídos os Institutos de Oncologia (que em Lisboa e no Porto asseguram duas das quatro unidades de Oncologia Pediátrica nacionais, e onde se realizam quase todos os transplantes pediátricos de células progenitoras hematopoiéticas).

Nas restantes categorias a maior parte dos internamentos ocorreu em hospitais do grupo III, excetuando-se as neonatais - aqui a distribuição pelos grupos I a III foi mais equilibrada, provavelmente refletindo nascimentos não programados ou com problemas inesperados (consistente com a maior percentagem de admissões urgentes nesta categoria). Os episódios neonatais foram os mais prolongados e onerosos, o que é de esperar dadas as características destes internamentos, ocorrendo maioritariamente numa unidade de cuidados intensivos especializada e com múltiplos problemas médico-cirúrgicos.

As crianças com múltiplas DCC têm uma maior probabilidade de serem readmitidas, bem como uma maior probabilidade de morte. ${ }^{15,31,35}$ Se dependentes de tecnologia, a despesa associada é 3,5 vezes superior à de crianças com uma DCC e sem apoio tecnológico. ${ }^{18} \mathrm{Em}$ Portugal Continental, comparando os episódios sem DCC com aqueles com mais do que uma categoria de DCC, estes foram 2,3 vezes mais longos, 2,8 vezes mais dispendiosos e tiveram mortalidade 78 vezes superior. Nos EUA em 2006, a duração de internamento foi três vezes mais longa, os gastos 11 vezes maiores e a mortalidade 15 vezes mais alta. ${ }^{15}$ A diferença de despesa entre Portugal Continental e os EUA provavelmente deve-se aos custos da saúde neste país.

Um estudo anterior já havia indicado que em Portugal quase $80 \%$ das mortes pediátricas com DCC ocorrem no hospital, ${ }^{3}$ provavelmente por falta de respostas no domicílio e na comunidade. Muitos, se não a maioria, dos óbitos pediátricos hospitalares ocorrem nas unidades de cuidados intensivos, em geral com demasiada intervenção médica e escassa discussão e planeamento sobre cuidados e opções de fim de vida. ${ }^{36,37}$ Nestas unidades torna-se necessário melhorar as formas de comunicar e de decidir, que devem ser alinhadas com os objetivos da criança e família. ${ }^{38}$

A experiência de um internamento hospitalar raramente é positiva. Sabemos que o internamento de uma criança é disruptivo para a família ${ }^{39}$ e que a ocorrência de erros é mais provável (3 vs 1,3/100 altas) em crianças com doenças crónicas, aumentando com o seu número, a sua gravidade e a duração do internamento. ${ }^{40}$

Por outro lado, as famílias desejam ser envolvidas nas decisões e planeamento, assim como serem capacitadas para os cuidados aos seus filhos, numa perspetiva resiliente de normalização da vida diária. ${ }^{41}$ Programas de melhoria da qualidade em crianças medicamente complexas, focados na capacitação da família e na coordenação 
de cuidados, resultam em redução dos internamentos, da sua duração e da despesa, simultaneamente melhorando os resultados clínicos e a satisfação das famílias. ${ }^{42,43}$

Mas é igualmente necessário melhorar a educação dos profissionais, em particular dos internos de Pediatria, sobre o cuidado destas crianças. ${ }^{17}$ Também os serviços de adultos devem organizar-se, já que o aumento da sobrevida pode colocar a necessidade de transição de cuidados para os seus serviços, que na maioria das vezes não estão adequadamente preparados. ${ }^{44}$

Por fim, não podemos ficar alheios ao aumento da despesa de saúde, um problema internacional em busca de soluções. Uma delas parece ser a integração de cuidados paliativos pediátricos, já que a aplicação dos seus princípios de promoção da qualidade de vida não aumenta a despesa e pode até diminuí-la, em especial na fase de fim de vida ${ }^{45,46}$ ou quando prestados na comunidade, incluindo o domicílio. ${ }^{47,48}$

Por todos estes motivos, os hospitais que cuidam de crianças com DCC devem considerar a criação de programas de cuidados paliativos pediátricos, ${ }^{17,49-51} \mathrm{com}$ equipas multidisciplinares que prestem orientação e/ou cuidados tanto no internamento como no ambulatório (ou mesmo no domicílio), conforme recomendado em 2014 ao Ministério da Saúde por um grupo de peritos. ${ }^{23}$ Embora numa primeira impressão as famílias possam ter algum 'receio' destas equipas, não compreendendo a sua contribuição para os cuidados dos seus filhos, a verdade é que o seu envolvimento é valorizado. Em particular as famílias apreciam a atitude destes profissionais e o seu apoio prático, a gestão de caso, planeamento e continuidade de cuidados. ${ }^{52} \mathrm{~A}$ prova da sua eficácia e resultados é o seu número crescente em vários países (nos EUA eram já 112 em 2013, existentes em $69 \%$ dos serviços respondentes a um inquérito). ${ }^{53}$

\section{Pontos fortes}

O uso de uma base de dados hospitalar nacional, alimentada por dados de rotina (com um número residual de informação omissa nas variáveis de interesse deste estudo) e com um grande número de episódios, é uma forma relativamente fácil de estimar as necessidades. Além disso, permitirá uma comparação dentro de alguns anos, após a esperada implementação de serviços de cuidados paliativos pediátricos. Destacamos também a nossa opção pela aplicação de uma classificação robusta da população alvo, utilizada na sua primeira versão ${ }^{1}$ em estudos semeIhantes noutros países. ${ }^{15,17,18,29}$ No entanto, os autores da classificação alertam para que o aumento da sensibilidade da versão 2 na identificação de crianças com DCC possa representar uma diminuição da especificidade. ${ }^{16}$

\section{Limitações}

O nosso estudo apresenta seis limitações. Primeiro, não podemos garantir que todos os episódios tenham sido corretamente codificados, sendo o mais provável uma subestimação do número de episódios com DCC. Segundo, quando existiram transferências inter-hospitalares de crianças, foram contabilizados os episódios em ambos os hospitais. Optámos por não os excluir, por entendermos que cada um deles representa atividade na respetiva instituição hospitalar (embora se trate do mesmo episódio de doença). Terceiro e quarto, não nos foi possível atribuir o local de internamento hospitalar (enfermaria ou unidade de cuidados intensivos) nem avaliar a gravidade ou a fase da doença. O estudo destes dois aspetos aprofundaria o conhecimento dos padrões nacionais de utilização hospitalar pelas crianças com DCC. Por exemplo, em Inglaterra as crianças com doenças limitantes da vida são responsáveis por $57,6 \%$ das admissões e $72,9 \%$ dos óbitos nas unidades de cuidados intensivos pediátricas. ${ }^{54}$ Quinto, dada a anonimização, não é possível ligar esta base a outras, o que poderia enriquecer a análise do ponto de vista sociodemográfico. Sexto, não contabilizámos os custos indiretos associados ao internamento (p.e. gastos adicionais da família, perda de rendimentos, faltas ao trabalho e escola).

\section{CONCLUSÃO}

Este estudo permite-nos concluir que em Portugal Continental as crianças medicamente complexas, com necessidades paliativas, representam uma parte muito importante da atividade e despesa do internamento hospitalar pediátrico. Esta noção é essencial para o planeamento de cuidados que se desejam eficazes, coordenados e sustentáveis, ${ }^{4,16}$ contribuindo para que os decisores políticos e clínicos possam tomar resoluções informadas e capazes de ir ao encontro das reais necessidades, atuais e futuras, de utentes e profissionais do SNS. Devemos ainda realçar que a prevalência crescente das DCC representa uma pressão sobre os recursos e o financiamento do sistema de saúde. ${ }^{6}$

O bem-estar das crianças com DCC e das suas famílias tem de ser uma preocupação de toda a sociedade. ${ }^{5}$ Elas exigem um modelo de cuidados adaptado às suas necessidades, e não o tradicional modelo reativo à doença aguda. ${ }^{4}$

Sendo utilizadoras intensivas dos recursos do SNS, estas crianças devem ser um alvo prioritário das reformas da coordenação e planeamento antecipado de cuidados (na saúde, educação e segurança social), ${ }^{35}$ tal como preconizado pelos princípios básicos dos cuidados paliativos pediátricos. ${ }^{19}$ Investigadores futuros deverão estudar os aspetos psicossociais, nomeadamente as atitudes, valores e preferências das crianças com DCC e suas famílias em Portugal.

Famílias que se sentem bem no seu dia-a-dia, envolvidas, suportadas e orientadas em cuidados complexos, são famílias que consomem menos serviços. O desenvolvimento de modelos de cuidados orientados para a criança com DCC e para o bem-estar familiar é uma obrigação atual da Pediatria, ${ }^{5}$ sendo imperativa uma reflexão dos responsáveis pelos serviços acerca das necessidades dos seus utentes.

\section{AGRADECIMENTOS}

Os autores agradecem à Administração Central do Sistema de Saúde (ACSS) pela cedência dos dados. 


\section{PROTECÇÃO DE PESSOAS E ANIMAIS}

Os autores declaram que os procedimentos seguidos estavam de acordo com os regulamentos estabelecidos pelos responsáveis da Comissão de Investigação Clínica e Ética e de acordo com a Declaração de Helsínquia da Associação Médica Mundial.

\section{CONFIDENCIALIDADE DOS DADOS}

Os autores declaram ter seguido os protocolos do seu centro de trabalho acerca da publicação de dados.

\section{REFERÊNCIAS}

1. Feudtner C, Hays RM, Haynes G, Geyer JR, Neff JM, Koepsell TD. Deaths attributed to pediatric complex chronic conditions: national trends and implications for supportive care services. Pediatrics. 2001;107:e99.

2. Fraser LK, Miller M, Hain R, Norman P, Aldridge J, McKinney PA, et al. Rising national prevalence of life-limiting conditions in children in England. Pediatrics. 2012;129:e923-9.

3. Forjaz de Lacerda A, Gomes B. Trends in cause and place of death for children in Portugal (a European country with no paediatric palliative care) during 1987-2011: a population-based study. BMC Pediatr. 2017; $17: 375$.

4. Wolfe I. Health services for children with long-term conditions and noncommunicable disease. In: Wolfe I, McKee M, editores. European child health services and systems: lessons without borders. Maidenhead: Open University Press; 2013. p. 63-92.

5. Boat TF, Filigno S, Amin RS. Wellness for families of children with chronic health disorders. JAMA Pediatr. 2017;171:825.

6. Crowley R, Wolfe I. Transition from children's to adults' services. In: Wolfe I, McKee M, editors. European child health services and systems: lessons without borders. Maidenhead: Open University Press; 2013. p. 93-112.

7. Wolfe I, Tamburlini G, Thompson M, Gill P, McKee M. Comprehensive strategies for improving child health services in Europe. In: Wolfe I, McKee M, editors. European child health services and systems: lessons without borders. Maidenhead: Open University Press; 2013. p. 251-2.

8. Cellarius V. The third wave of palliative care. J Pall Care. 2014;30:287290.

9. Assembleia da Organização Mundial de Saúde. Strengthening of palliative care as a component of comprehensive care throughout the life course. 2014. [consultado 2018 fev 8]. Disponível em: http://apps. who.int/medicinedocs/documents/s21454en/s21454en.pdf.

10. Higginson IJ, Hart S, Koffman J, Selman L, Harding R. Needs assessments in palliative care: an appraisal of definitions and approaches used. J Pain Symptom Manage. 2007;33:500-5.

11. Feudtner $C$, Christakis DA, Connell FA. Pediatric deaths attributable to complex chronic conditions: a population-based study of Washington State, 1980-1997. Pediatrics. 2000;106:205.

12. Pousset G, Bilsen J, Cohen J, Addington-Hall J, Miccinesi G, OnwuteakaPhilipsen B, et al. Deaths of children occurring at home in six European countries. Child Care Health Dev. 2010;36:375-84.

13. Feudtner C, Christakis DA, Zimmerman FJ, Muldoon JH, Neff JM, Koepsell TD. Characteristics of deaths occurring in children's hospitals: implications for supportive care services. Pediatrics. 2002;109:887-93.

14. Feudtner C, DiGiuseppe DL, Neff JM. Hospital care for children and young adults in the last year of life: a population-based study. BMC Med. 2003;1:3.

15. Simon TD, Berry J, Feudtner C, Stone BL, Sheng X, Bratton SL, et al. Children with complex chronic conditions in inpatient hospital settings in the United States. Pediatrics. 2010;126:647-55.

16. Feudtner C, Feinstein JA, Zhong W, Hall M, Dai D. Pediatric complex chronic conditions classification system version 2: updated for ICD-10 and complex medical technology dependence and transplantation. BMC Pediatr. 2014;14:199.

17. Burns KH, Casey PH, Lyle RE, Bird TM, Fussell JJ, Robbins JM. Increasing prevalence of medically complex children in US hospitals. Pediatrics. 2010;126:638-46.

18. Cohen E, Berry JG, Camacho X, Anderson G, Wodchis W, Guttmann A. Patterns and costs of health care use of children with medical complexity. Pediatrics. 2012;130:e1463-70.

19. Organização Mundial de Saúde. Definition of pediatric palliative care. 1998. [consultado 2018 fev 8]. Disponível em http://www.who.int/cancer/ palliative/definition/en/.

\section{CONFLITO DE INTERESSES}

Os autores declaram não ter nenhum conflito de interesses na realização do presente artigo.

\section{FONTES DE FINANCIAMENTO}

Os autores declaram não ter recebido subsídios ou bolsas para a elaboração do artigo.

20. Quinn C, McCarthy S, Devins M, O'Reilly M, Twomey M, Ling J. Prioritisation of future research topics in paediatric palliative care in Ireland: a Delphi study. Int J Pall Nurs. 2017;23:88.

21. Craft A, Killen S. Palliative care services for children and young people in England. An independent review for the Secretary of State of Health. Londres. 2007. [consultado 2013 nov 26]. Disponível em http://webarchive.nationalarchives.gov.uk/20080817153114/http://www dh.gov.uk/en/Publicationsandstatistics/Publications/PublicationsPolicy AndGuidance/DH_074459.

22. International Children's Palliative Care Network. Levels of provision of children's palliative care. [consultado 2013 nov 26]. Disponível em http:// www.icpcn.org/1949-2/.

23. Grupo de Trabalho do Gabinete do Secretário de Estado Adjunto do Ministro da Saúde. Cuidados Paliativos Pediátricos. Lisboa; 2014. [consultado 2018 fev 8]. Disponível em http://www.apcp.com.pt/uploads/ Relato-rio-do-GdT-de-CPP.pdf.

24. Downing J, Knapp C, Muckaden MA, Fowler-Kerry S, Marston J, on behalf of the ICPCN Scientific Committee. Priorities for global research into children's palliative care: results of an International Delphi Study. BMC Palliat Care. 2015;14:3.

25. Fraser L, Jarvis S, Moran N, Aldridge J, Parslow R, Beresford B. Children in Scotland requiring palliative care: identifying numbers and needs (The ChiSP Study). York; 2015. A report commissioned by the Childrens Hospice Association Scotland. [consultado 2018 fev 8]. Disponível em http://www.cen.scot.nhs.uk/wp-content/uploads/sites/24/2017/02/ ChiSP_report.pdf.

26. Despacho 9871/2010. Diário da República, $2^{\mathrm{a}}$ série, $\mathrm{n}^{\circ} 112-11 \mathrm{de}$ Junho de 2010. [consultado 2018 fev 6]. Disponível em https://dre. tretas.org/dre/275684/despacho-9871-2010-de-11-de-junho.

27. Instituto Nacional de Estatística. NUTS 2013 - as Novas Unidades Territoriais Para Fins Estatísticos. Lisboa; 2015. [consultado 2018 fev 6]. Disponível em https://www.ine.pt/xportal/xmain?xpid=INE\&xpgid=ine publicacoes\&PUBLICACOESpub_boui=230205992\&PUBLICACOES modo $=2 \&$ xlang $=$ pt.

28. Martins R, Salgado C, Espirito Santo R, Pereira C, Araújo e Sá G, Machado MC. Casuística do internamento pediátrico de curta duração de um hospital terciário: uma nova realidade? Acta Pediatr Port. 2017;48:137-46.

29. Ananth P, Melvin P, Feudtner C, Wolfe J, Berry JG. Hospital use in the last year of life for children with life-threatening complex chronic conditions. Pediatrics. 2015;136:938-46.

30. Berry JG, Hall DE, Kuo DZ, Cohen E, Agrawal R, Feudtner C, et al. Hospital utilization and characteristics of patients experiencing recurrent readmissions within children's hospitals. JAMA. 2011;305:682-90.

31. Feudtner C, Pati S, Goodman DM, Kahn MG, Sharma V, Hutto JH, et al. State-level child health system performance and the likelihood of readmission to children's hospitals. J Pediatr. 2010;157:98-102.

32. Mesman GR, Kuo DZ, Carroll JL, Ward WL. The impact of technology dependence on children and their families. J Pediatr Health Care 2013;27:451-9.

33. Coller RJ, Nelson BB, Sklansky DJ, Saenz AA, Klitzner TS, Lerner CF, et al. Preventing hospitalizations in children with medical complexity: a systematic review. Pediatrics. 2014;134:e1628-47.

34. Frierdich S, Goes C, Dadd G. Community and home care services provided to children with cancer: a report from the Children's Cancer Group Nursing Committee-Clinical Practice Group. J Pediatr Oncol Nurs. 2003;20:252-9.

35. Feudtner C, Levin JE, Srivastava R, Goodman DM, Slonim AD, Sharma $\mathrm{V}$, et al. How well can hospital readmission be predicted in a cohort 
of hospitalized children? A retrospective, multicenter study. Pediatrics. 2009;123:286-93.

36. McCallum DE, Byrne P, Bruera E. How children die in hospital. J Pain Symptom Manage. 2000;20:417-23.

37. Blume ED, Balkin EM, Aiyagari R, Ziniel S, Beke DM, Thiagarajan R, et al. Parental perspectives on suffering and quality of life at end-oflife in children with advanced heart disease. Pediatr Crit Care Med. 2014;15:336-42.

38. Marcus KL, Henderson CM, Boss RD. Chronic critical illness in infants and children. Pediatr Crit Care Med. 2016;17:743-52.

39. Adams S, Cohen E, Mahant S, Friedman JN, MacCulloch R, Nicholas DB. Exploring the usefulness of comprehensive care plans for children with medical complexity (CMC):a qualitative study. BMC Pediatr. 2013;13:10.

40. Ahuja N, Zhao W, Xiang H. Medical errors in US pediatric inpatients with chronic conditions. Pediatrics. 2012;130:e786-93.

41. Leyenaar JK, O'Brien ER, Leslie LK, Lindenauer PK, Mangione-Smith R. Families' priorities regarding hospital to home transitions for children with medical complexity. Pediatrics. 2017;139:e20161581.

42. Noritz G, Madden M, Roldan D, Wheeler TA, Conkol K, Brilli RJ, et al. A population intervention to improve outcomes in children with medical complexity. Pediatrics. 2017;139:e20153076.

43. Petitgout JM, Pelzer DE, McConkey SA, Hanrahan K. Development of a hospital-based care coordination program for children with special health care needs. J Pediatr Health Care. 2013;27:419-25.

44. Doug M, Adi Y, Williams J, Paul M, Kelly D, Petchey R, et al. Transition to adult services for children and young people with palliative care needs: a systematic review. Arch Dis Child. 2011;96:78-84.

45. Smith AG, Andrews S, Bratton SL, Sheetz J, Feudtner C, Zhong W, et al. Pediatric palliative care and inpatient hospital costs: a longitudinal cohort study. Pediatrics. 2015;135:694-700
46. Postier A, Chrastek J, Nugent S, Osenga K, Friedrichsdorf SJ. Exposure to home-based pediatric palliative and hospice care and its impact on hospital and emergency care charges at a single institution. J Pall Med. 2013;17:131231061544001.

47. Gans D, Hadler MW, Chen X, Shang-Hua W, Dimand R, Abramson JM et al. Cost analysis and policy implications of a pediatric palliative care program. J Pain Symptom Manage. 2016;52:329-35.

48. Chong PH, De Castro Molina JA, Teo K, Tan WS. Paediatric palliative care improves patient outcomes and reduces healthcare costs: evaluation of a home-based program. BMC Palliat Care. 2018;17:966.

49. Committee on Bioethics and Committee on Hospital Care, American Academy of Pediatrics. Palliative care for children. Pediatrics. 2000;106:351-7.

50. Steering Committee of the EAPC task force on palliative care for children and adolescents. IMPaCCT: standards for paediatric palliative care in Europe. Eur J Palliat Care. 2007;14:109-14.

51. Section on Hospice and Palliative Medicine and Committee on Hospital Care, American Academy of Pediatrics. Pediatric palliative care and hospice care commitments, guidelines, and recommendations. Pediatrics. 2013;132:966-72.

52. Verberne LM, Schouten-van Meeteren AY, Bosman DK, Colenbrander DA, Jagt CT, Grootenhuis MA, et al. Parental experiences with a paediatric palliative care team: a qualitative study. Pall Med. 2017;21:026921631769268.

53. Feudtner C, Womer J, Augustin R, Remke S, Wolfe J, Friebert S et al. Pediatric palliative care programs in children's hospitals: a crosssectional national survey. Pediatrics. 2013;132:1-8.

54. Fraser LK, Parslow R. Children with life-limiting conditions in paediatric intensive care units: a national cohort, data linkage study. Arch Dis Child. Arch Dis Child. 2018:103:540-7. 